\title{
Relación longitud-peso y factor de condición de los peces nativos del río San Pedro (cuenca del río Valdivia, Chile)
}

\section{Weight-length relationships and condition factor of native fish from San Pedro River (Valdivia River basin, Chile)}

\author{
Roberto Cifuentes ${ }^{1,2}$, Jorge González ${ }^{1}$, Germán Montoya ${ }^{1}$, Alfonso Jara ${ }^{1}$, Néstor Ortiz ${ }^{1}$, Priscila \\ PIEDRA $^{1}$ \& EVELYN HABIT ${ }^{1}$
}

${ }^{1}$ Laboratorio de Ecología y Conservación de Peces de Aguas Continentales, Unidad de Sistemas Acuáticos, Centro EULA, Universidad de Concepción. Casilla 160-C

${ }^{2}$ Departamento de Zoología, Facultad de Ciencias Naturales y Oceanográficas, Universidad de Concepción.

*EMAIL: roberto.cifuentes@gmail.com

\begin{abstract}
RESUMEN
La relación longitud-peso y el factor de condición $(K)$ son descriptores de gran interés en la biología de poblaciones de peces, ya que aportan información fundamental sobre estrategias de crecimiento, estado nutricional y reproducción. También son parámetros ampliamente utilizados para comparar la condición de poblaciones que habitan en sistemas acuáticos con distintos grados de intervención antrópica. Por ello, conocer el comportamiento de poblaciones que habitan en ecosistemas prístinos, entrega información de base, relevante para comprender cambios o efectos en poblaciones sometidas a estas presiones. El río San Pedro de la cuenca del Valdivia, sur de Chile, es un sistema que se caracteriza por su actual bajo nivel de intervención, su alta diversidad íctica y baja abundancia de especies introducidas. En este trabajo describimos la relación longitud-peso y factor de condición de 12 especies de peces nativos del río San Pedro, durante más dos ciclos anuales, analizando sus estrategias de crecimiento y variabilidad estacional del factor de condición. Los resultados de la relación longitud-peso muestran que gran parte de las especies presentan un crecimiento isométrico, especialmente Diplomystes camposensis, Basilichthys australis y Brachygalaxias bullocki. Sin embargo, algunas especies presentan una tendencia a la alometría (Galaxias maculatus, Galaxias platei y Trichomycterus areolatus). Los valores de $K$ muestran que especies como Percilia gillissi poseen una marcada variabilidad anual, posiblemente asociada a las épocas reproductivas y de mayor oferta de alimento. Entregamos esta información como base para entender el funcionamiento de poblaciones ícticas en condiciones naturales y para ser utilizada en la evaluación de futuros proyectos que puedan impactar estos ecosistemas.
\end{abstract}

Palabras clave: Río San Pedro, relación longitud - peso, K, peces nativos.

\begin{abstract}
The length-weight relationship and condition factor $(K)$ are useful descriptors in population fish biology, because they provide essential information on growth strategies, nutritional status and reproduction. They are also parameters widely used to compare the condition of aquatic systems with varying degrees of human intervention. Therefore, understanding the dynamics of populations in pristine ecosystems, provides relevant basic information to understanding changes or effects in populations under these pressures. The San Pedro river (Valdivia basin), southern Chile, is a system characterized by its current low intervention level, high fish diversity and low abundance of introduced species. We describe the length-weight relationship and condition factor of 12 native fish of the San Pedro river, for over two annual cycles, analyzing their growth strategies and seasonal variability in condition factor. The results of the length-weight show that the most of species exhibit isometric growth, especially Diplomystes camposensis, Basilichthys australis and Brachygalaxias bullocki, however, some species have a tendency to allometry (Galaxias maculatus, Galaxias platei and Trichomycterus areolatus). The values of $K$ show that species such as Percilia gillissi have a strong annual variability, associated with the breeding season and greater supply of food. We give this information as baseline data to understand the functioning of fish populations in natural conditions and to be used for the evaluation of future projects that may impact these ecosystems.
\end{abstract}

Keywords: San Pedro river, weight-length relationship, $K$, native fish. 


\section{INTRODUCCIÓN}

El estudio de las relaciones longitud-peso y los índices de condición en peces proporcionan información indirecta sobre el crecimiento, madurez, reproducción, nutrición y por ende del estado de salud de las poblaciones. Ello permite efectuar estudios comparativos interpoblacionales (Granado 1996; Arismendi et al. 2011) que luego pueden ser usados en modelos predictivos del comportamiento o dinámica, tanto de la población como de la comunidad (McCallum 2000). De tal modo que, conocer tales características en poblaciones de peces que habitan sistemas con baja o nula intervención antrópica es fundamental para predecir potenciales cambios en poblaciones amenazadas o que habitan sistemas perturbados. En este sentido, las relaciones longitud-peso son utilizadas con frecuencia para modelar las tendencias de la biomasa basados en la talla y son ampliamente usados en el manejo de poblaciones de importancia económica (Treer et al. 2008; Agboola \& Anetekhai 2008). Así también, diferentes autores (Carlander 1969; Froese 2006) han evidenciado que la mayoría de las especies ( $>90 \%)$ poseen un crecimiento isométrico evidenciado por una tendencia general al aumento del grosor, proporcional a la talla, durante el crecimiento. En general, las especies que presentan valores fuera de este rango se deben a muestras no representativas numéricamente o a especies particulares que presentan cambios morfológicos notables durante su ontogenia (Froese 2006). Estos cambios podrían estar asociados a rasgos adaptativos con alto valor evolutivo. Por su parte, el factor de condición, comúnmente designado como K, es utilizado para comparar la "condición" o "bienestar" de un pez o población, basándose en que los peces de mayor peso, a una determinada longitud, presentan una mejor condición (Froese 2006). No obstante, la interpretación de los índices de condición debe hacerse cuidadosamente pues pueden depender de varios factores (Froese 2006; McPherson et al. 2011), e.g. disponibilidad de alimento (Rennie \& Verdon 2008) o estacionalidad (Blackwell et al. 2000; Trudel et al. 2005), incluyendo su interrelación (Rennie et al. 2008).

En este trabajo describimos y analizamos la relación longitud-peso y el factor de condición de especies nativas presentes en el río San Pedro (cuenca del río Valdivia), el cual se caracteriza por ser uno de los pocos sistemas fluviales actualmente prístino. Esta información constituye una referencia útil para conocer las relaciones y tendencias esperadas de longitud y peso de las especies estudiadas en condiciones naturales.

\section{METODOLOGÍA}

Área de Estudio y Diseño de Muestreo

El estudio fue llevado a cabo en el río San Pedro, tributario del río Valdivia, al sur de Chile. Este río tiene una extensión de $40 \mathrm{~km}$ desde su origen en el desagüe del Lago Riñihue (39 $\left.46^{\prime} \mathrm{S} ; 7^{\circ} 27^{\prime} \mathrm{W}\right)$, hasta su confluencia con el río Quinchilca (39 $\left.51^{\prime} \mathrm{S} ; 7^{\circ} 27^{\prime} \mathrm{W}\right)$, donde cambia de nombre a río Calle-Calle. El régimen de caudal de este río es pluvial con un máximo en julio, precedido por bajos caudales entre febrero a mayo.

Se establecieron 35 sitios de muestreo en el río, abarcando la totalidad de su recorrido. El estudio se realizó entre octubre de 2005 y marzo de 2008, efectuando un total de 465 días efectivos de muestreo, utilizando pesca eléctrica (Smith Root LR-24) en zonas ribereñas del río y redes monofilamento en áreas asociadas al desagüe del lago Riñihue. Cada individuo capturado fue anestesiado mediante solución anestésica diluida en agua (BZ-20®, $1 \mathrm{~mL} / 5 \mathrm{~L})$, medido con ictiómetro $(0,1 \mathrm{~cm}$ de precisión) y pesado mediante balanza digital, de $400 \mathrm{~g}$ (precisión $0,01 \mathrm{~g}$ ) o 2000 $\mathrm{g}$ (precisión $0,1 \mathrm{~g}$ ), dependiendo del peso del pez. Una vez recuperada su movilidad, todos los peces fueron liberados a su hábitat de origen. La medición de las tallas fue longitud estándar (LE).

\section{ANÁLISIS DE DATOS}

Se analizaron las relaciones de longitud-peso por especie mediante regresión lineal, calculando los valores de $a$ y $b$ de la ecuación $W=a L^{b}$ (Froese 2006), donde $W$ es el peso total en gramos y $L$ la longitud en $\mathrm{cm}$. Debido a que la longitud es una magnitud lineal y el peso es igual al cubo de la talla, si un individuo mantiene su forma al crecer, entonces el crecimiento es isométrico $(b=3)$. Cuando $b>3$, los individuos de mayor talla han incrementado su peso en mayor proporción que su longitud, presentando crecimiento alométrico positivo. En cambio, cuando $b<3$, los individuos incrementan preferencialmente su longitud relativa más que su peso. Se consideraron especies de crecimiento isométrico las que fluctuaron dentro de los valores $b=2,5$ y $b=3,5$ (sensu Carlander 1969; Froese 2006). El estado de condición por individuo se estimó mediante el índice de Fulton $(K)$ (Ricker 1975); $K=100\left(W / L^{3}\right)$, donde $W$ es el peso corporal húmedo en gramos y $L$ la longitud en cm. Luego se analizó la variación anual de $K$ por especie mediante un test ANOVA de una vía analizando los datos por meses. Con el fin de estimar las longitudes totales de los individuos se elaboraron ecuaciones de ajuste para cada especie mediante regresión lineal. Esto se realizó en base a datos de longitud estándar (LE) y total (LT) de 50-60 individuos por especie almacenados en el Laboratorio de Ecología y Conservación de Peces del Centro EULA (Universidad de Concepción, Chile).

\section{RESULTADOS}

Durante el período de estudio se capturaron 31.022 individuos distribuidos en 12 especies. Los principales 
parámetros descriptivos de las poblaciones (número de individuos- $N$, longitud máxima y mínima, promedio $\pm \mathrm{DS}$, moda, coeficiente de crecimiento isométrico $(b)$, relación longitud-peso) se entregan en la Tabla 1. La distribución de frecuencias de talla (Fig. 1) muestra que especies con $N$ altos presentaron tallas comprendidas dentro del rango que abarca sus estados juveniles y adultos (i.e. Percilia gillissi Girard 1854, Galaxias maculatus (Jenyns 1842), Galaxias platei Steindachner 1898, Trichomycterus areolatus Valenciennes 1846, Diplomystes camposensis Arratia 1987, Cheirodon australe Eigenmann 1927 y Brachygalaxias bullocki Regan 1908). En cambio especies como Basilichthys australis Eigenmann 1927, Odontesthes mauleannum (Steindachner 1896) y Geotria australis Gray
1851, estuvieron representadas principalmente por estados larvales y juveniles. De hecho, a pesar de su elevado $N$, no se registraron adultos para esta última especie.

Los valores del coeficiente de crecimiento $b$ muestran que la mayoría de las especies presentan un desarrollo comprendido dentro del rango isométrico (Tabla 1; Fig. 2). Sólo $G$. australis $(b=2,11)$ presentó un crecimiento alométrico negativo. Sin embargo, algunas especies presentaron una tendencia a la alometría positiva con valores de $b>3,15$ (G. maculatus, G. platei y Aplochiton taeniatus), y otras, tendencia a la alometría negativa con valores $b<2,87$ ( $T$. areolatus, Cheirodon australe, P. gillissi y Percicthys trucha).

TABla 1. Número total de individuos, tallas máximas y mínimas, y parámetros de la relación entre longitud y peso ( $a$ y $b$ ) para 12 especies nativas presentes del río San Pedro; $r$ es el coeficiente de correlación en el que todas las especies resultaron altamente significativas $P<0,05$. El parámetro $b$ en esta tabla representa la forma de crecimiento para cada población: isométrico $(b=3)$, alométrico positivo $(b>3)$, alométrico negativo $(b<3)$.

TABLE 1. Total number of individuals, maximum and minimum sizes, and parameters of the relationship between length and weight $(a$ and $b$ ) for 12 native species from San Pedro River; $r$ is the correlation coefficient, which all species were highly significant $P<0.05$. The parameter $b$ represents the shape of growth for each population: isometric $(b=3)$, positive allometry $(b>3)$, negative allometry $(b<3)$.

\begin{tabular}{|c|c|c|c|c|c|c|c|c|}
\hline \multirow[b]{2}{*}{ Especie } & \multirow[b]{2}{*}{$\mathrm{N}$} & \multicolumn{4}{|c|}{ Longitud Estándar (cm) } & \multicolumn{3}{|c|}{$\mathrm{W}=a \mathrm{~L} b$} \\
\hline & & Min. & Max. & $\begin{array}{c}\text { Promedio } \pm \\
\text { DE }\end{array}$ & Moda & $a$ & $b$ & $\begin{array}{c}r \\
* * p<<0,01\end{array}$ \\
\hline Percilia gillissi Girard 1854 & 7898 & 1,1 & 8 & $4,0 \pm 0,96$ & 4 & 0,021 & 2,87 & $0,958 * *$ \\
\hline Galaxias maculatus (Jenyns 1842) & 6956 & 0,8 & 9,7 & $5,2 \pm 0,96$ & 5,2 & 0,003 & 3,39 & $0,923 * *$ \\
\hline Galaxias platei Steindachner 1898 & 4521 & 2,1 & 16,8 & $5,5 \pm 2,24$ & 4 & 0,005 & 3,15 & $0,978^{* *}$ \\
\hline Basilichthys australis Eigenmann 1927 & 2733 & 0,7 & 36,5 & $4,0 \pm 2,78$ & 0.9 & 0,006 & 2,94 & $0,978 * *$ \\
\hline Trichomycterus areolatus Valenciennes 1846 & 3797 & 0,3 & 17,6 & $4,5 \pm 1,85$ & 3,3 & 0,015 & 2,61 & $0,943 * *$ \\
\hline Cheirodon australe Eigenmann 1927 & 1500 & 0,9 & 6,5 & $3,3 \pm 0,97$ & 4 & 0,017 & 2,78 & $0,941 * *$ \\
\hline Geotria australis Gray 1851 & 1007 & 1,1 & 11,4 & $8,5 \pm 1,27$ & 8,5 & 0,011 & 2,11 & $0,874 * *$ \\
\hline Diplomystes camposensis Arratia 1987 & 900 & 1,5 & 22 & $5,4 \pm 3,1$ & 4,2 & 0,009 & 3,03 & $0,981 * *$ \\
\hline Percicthys trucha Valenciennes 1833 & 667 & 1,3 & 36 & $6,3 \pm 2,83$ & 7 & 0,018 & 2,83 & $0,988 * *$ \\
\hline Brachygalaxias bullocki Regan 1908 & 623 & 1,8 & 4,6 & $2,9 \pm 0,6$ & 3,1 & 0,029 & 2,93 & $0,936^{* *}$ \\
\hline Aplochiton taeniatus Jenyns 1842 & 329 & 2,3 & 9,8 & $5,6 \pm 0,94$ & 5,5 & 0,004 & 3,38 & $0,938^{* *}$ \\
\hline $\begin{array}{l}\text { Odontesthes mauleannum (Steindachner } \\
\text { 1896) }\end{array}$ & 91 & 2,4 & 30,5 & $7,4 \pm 5,3$ & 3,2 & 0,011 & 2,93 & $0,996 * *$ \\
\hline
\end{tabular}


Gayana 75(2), 2012

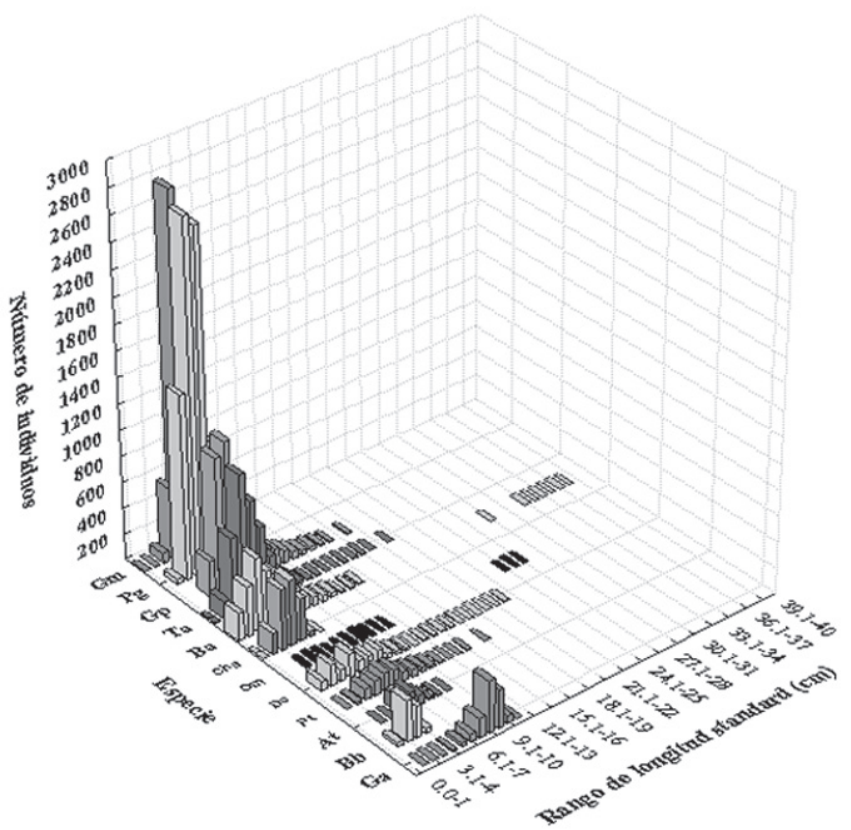

Figura 1. Distribución de frecuencias de talla para 12 especies nativas del río San Pedro, por rango de longitud estándar. At=Aplochiton taeniatus; $\mathrm{Bb}=$ Brachygalaxias bullocki; $\mathrm{Ba}=$ Basilichthys australis; $\mathrm{Ca}=$ Cheirodon australe $; \mathrm{Dc}=$ Diplomystes camposensis; $\mathrm{Gm}=$ Galaxias maculatus; $\mathrm{Gp}=$ Galaxias platei; $\mathrm{Ga}=$ Geotria australis; $\mathrm{Pg}=$ Percilia gillisi; $\mathrm{Pt}=$ Percicthys trucha; Om= Odontesthes mauleanum $\mathrm{y}$ $\mathrm{Ta}=$ Trichomycterus areolatus.

Figure 1. Size frequency distribution for 12 native species from San Pedro river, by standard length. At=Aplochiton taeniatus; $\mathrm{Bb}=$ Brachygalaxias bullocki; $\mathrm{Ba}=$ Basilichthys australis; $\mathrm{Ca}=$ Cheirodon australe; $\mathrm{Dc}=$ Diplomystes camposensis; $\mathrm{Gm}=$ Galaxias maculatus; $\mathrm{Gp}=$ Galaxias platei; $\mathrm{Ga}=$ Geotria australis; $\mathrm{Pg}=$ Percilia gillisi; $\mathrm{Pt}=$ Percicthys trucha; $\mathrm{Om}=$ Odontesthes mauleanum and $\mathrm{Ta}=$ Trichomycterus areolatus.

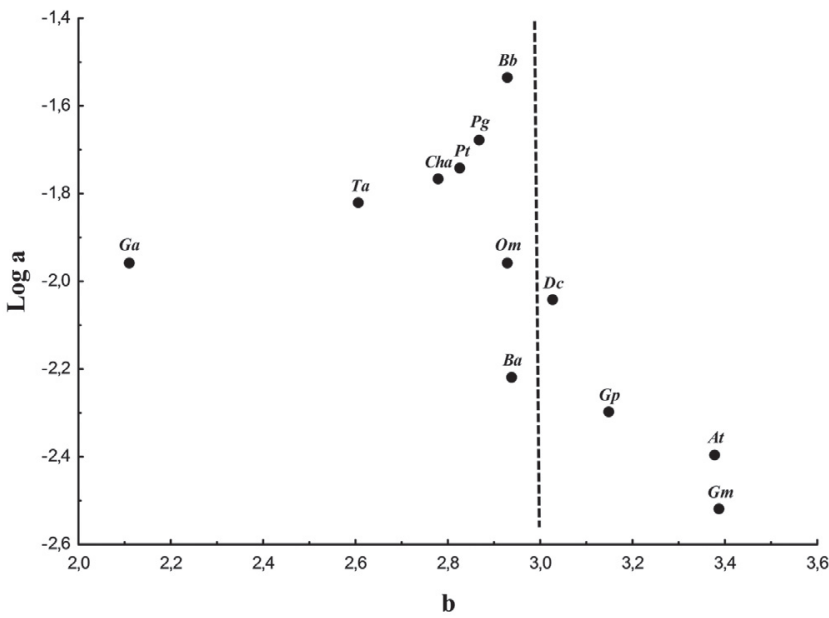

Figura 2. Relación entre los parámetros $b$ y $\log a$ de 12 especies nativas del río San Pedro. Se observa la distribución de las especies en relación a un crecimiento isométrico $(\mathrm{b}=3)$. At $=$ Aplochiton taeniatus; $\mathrm{Bb}=$ Brachygalaxias bullocki; $\mathrm{Ba}=$ Basilichthys australis; $\mathrm{Ca}=$ Cheirodon australe $; \mathrm{Dc}=$ Diplomystes camposensis $; \mathrm{Gm}=$ Galaxias maculatus $; \mathrm{Gp}=$ Galaxias platei $\mathrm{Ga}=$ Geotria australis; $\mathrm{Pg}=$ Percilia gillisi; $\mathrm{Pt}=$ Percicthys trucha; $\mathrm{Om}=$ Odontesthes mauleanum y Ta=Trichomycterus areolatus. Galáxidos como $\mathrm{Gp}, \mathrm{Gm}$ y At $(b>3)$ tienden a presentar un crecimiento alométrico positivo. Por otra parte, Ta presenta una tendencia hacia la alometría negativa $(b<3)$.

FiguRE 2. Relationships between $b$ and $\log a$ parameters of 12 native species from San Pedro river. It shows the species distribution in relation to an isometric growth; $b=3$ (grey line). $\mathrm{At}=$ Aplochiton taeniatus; $\mathrm{Bb}=$ Brachygalaxias bullocki; $\mathrm{Ba}=$ Basilichthys australis; $\mathrm{Ca}=$ Cheirodon australe $; \mathrm{Dc}=$ Diplomystes camposensis $; \mathrm{Gm}=$ Galaxias maculatus $; \mathrm{Gp}=$ Galaxias platei $; \mathrm{Ga}=$ Geotria australis $; \mathrm{Pg}=$ Percilia gillisi; $\mathrm{Pt}=$ Percicthys trucha; $\mathrm{Om}=$ Odontesthes mauleanum and Ta=Trichomycterus areolatus. Galaxids as $\mathrm{Gp}, \mathrm{Gm}$ and $\mathrm{At}(b>3)$ tend to positive alometric growth, while Ta tend to negative alometric growth $(b<3)$. 
En cuanto a $K$ (Fig. 3), la variación del patrón observado difiere entre las especies. En este sentido, P. gillissi (Fig. 3a) evidencia un marcado patrón estacional con diferencias significativas en su variación intranual $\left(\right.$ ANOVAF $_{(11)}=124,4$; $P<0,01)$. Esta especie presenta dos máximos en el valor de $K$ durante el año, uno en invierno y otro en verano. El mismo patrón con diferencias significativas menos notables que en $P$. gillissi se observa en $G$. maculatus (ANOVA $\mathrm{F}_{(11)}=397,1 ; P<0,01$ ), y en Ch. australe (Fig. 3c) (ANOVA $\left.\mathrm{F}_{(11)}=18,34 ; P<0,01\right)$ (Fig. 3b y $3 \mathrm{c}$, respectivamente). Por otra parte, cuatro especies mostraron un patrón similar de variación anual con un único máximo en la época estival (enero a marzo), siendo la variación anual significativa (Fig. 3b, d, e y f, respectivamente). Estas corresponden a $G$. platei (ANOVA $\left.\mathrm{F}_{(10)}=11,35 ; P<0,01\right), B$. australis (ANOVA $\mathrm{F}_{(11)}=10,90 ; P<0,05$ ), A. taeniatus (ANOVA $\mathrm{F}_{(10)}=2,18$; $P<0,05$ ), $O$. mauleanum (ANOVA $\mathrm{F}_{(7)}=17,5 ; P<0,01$ ). $A$ taeniatus y $G$. platei están en máximo valor de $K$ en febrero, mostrando el resto del año una variación muy similar, coincidiendo en sus mínimos valores de $K$ en invierno. Basilichthys australis presenta su mínimo y máximo valor de $K$ en diciembre y enero respectivamente, disminuyendo bruscamente en febrero, manteniéndose el resto del año en valores más estables. Odontesthes mauleanum muestra su máximo valor en marzo, pero no fue posible establecer un patrón anual mas preciso debido a su ausencia en las muestras en varios meses. Otras especies mostraron una variación anual altamente significativa con un máximo durante invierno: $D$. camposensis (ANOVA $\left.\mathrm{F}_{(11)}=9,40 ; P<0,05\right), B$. bullocki (ANOVA $\left.\mathrm{F}_{(11)}=3,85 ; P<0,05\right)$ y $P$. trucha (ANOVA $\left.\mathrm{F}_{(11)}: 4,85 ; \mathrm{P}<0,01\right)$ (Fig. $3 \mathrm{c}$ y $3 \mathrm{f}$, respectivamente). A finales de invierno, T. areolatus (Fig. 3d) presentó una variabilidad anual menor respecto de las demás especies, aunque igualmente significativa (ANOVA $\mathrm{F}_{(11)}=5,93 ; P<0,05$ ). Para G. australis (Fig. 3e), la variación de $K$ durante el año fue igualmente significativa (ANOVA $\mathrm{F}_{(10)}=11,35 ; P<0,01$ ), pero sin una fluctuación marcada en el ciclo anual.

\section{DISCUSIÓN}

El estudio de la biología de las poblaciones de peces nativos de agua dulce de Chile es escaso (Habit et al. 2006), por lo que el presente trabajo entrega información inédita para la mayoría de las especies. Se encontró que gran parte de estas especies presentan un crecimiento del tipo isométrico. Por otra parte, se encontró una alta variación estacional en el factor de condición de las especies, lo que pone de manifiesto las distintas estrategias de alimentación y reproducción utilizadas por el ensamble de peces del río San Pedro.

RELACIÓN PESO-LONGITUD

Las especies B. bullocki, P. gillissi, D. camposensis, T. areolatus, Ch. australe, G. maculatus y $G$. platei son consideradas como especies de crecimiento isométrico, con valores entre $b=2,6$ y $b=3,4$. Estas especies mostraron gran representatividad de tallas, por lo cual estos valores evidencian una tendencia general al aumento del grosor proporcional a la talla durante el crecimiento. No obstante, se evidenció que algunas de estas especies (i.e. G. maculatus y $G$. platei) presentaron valores $b>3$, que estarían más acorde con un aumento progresivo de la robustez desde formas tempranas más elongadas o "delgadas", por lo cual hay una mayor ganancia proporcional en peso que en longitud, fenómeno que es común entre peces (sensu Froese 2006). Con respecto a los valores $b<3$ presentados principalmente por $T$. areolatus, seguido distantemente por Ch. australe, lo patrones de crecimiento se asocian a que los individuos de mayor talla son más elongados que los pequeños (sensu Froese 2006). Es interesante también que, especies de morfotipos similares, i.e. fusiforme en G. maculatus y $T$. areolatus muestran un tipo de crecimiento totalmente opuesto (Fig. 1), evidenciando notables diferencias entre las tallas máximas analizadas en relación a individuos más pequeños. Si bien los valores fuera del rango predicho por Carlander (1969) y Froese (2006), son frecuentemente producto de muestras poco representativas numéricamente (Froese 2006), la gran cantidad de datos analizados en este estudio para T. areolatus y G. maculatus $(n>2189)$ evidencian que dichas especies presentan un patrón de crecimiento alométrico que podría conllevar importantes ventajas adaptativas asociadas al uso del hábitat bentónico para T. areolatus. De hecho, Habit et al. (2005), relaciona los hábitos bentónicos de ésta especie con el gran éxito de sobrevivencia en diferentes ambientes tanto naturales como alterados. En tanto que para G. maculatus, podría tener importantes efectos en las diferentes estrategias de historia demostradas (Chapman et al. 2006). Diplomystes camposensis evidencia un patrón de crecimiento isométrico, al igual que la especie congenérica $D$. nahuelbutaensis Arratia, 1987 (Vila et al. 1996; Habit 2005).

\section{FACTOR DE CONDICIÓN}

En particular, las fechas con bajos $K$ observados para $G$. maculatus (mostrando bruscas bajas en enero y luego en marzo), coinciden con los hallazgos de huevos reportados por Montoya et al. (2012) en este mismo río y época, por lo cual tales bajas indicarían una época de desove para esta especie (Fig. 3b). Las alzas de $K$ entre junio-septiembre (Fig. $3 b)$ estarían asociadas con un segundo período reproductivo, evidenciado por la presencia de hembras maduras durante julio-septiembre en este mismo río (Montoya et al. 2012), aunque no es descartable que esté asociado a las alzas de caudal en julio que propician una mayor disponibilidad de alimento en planicies de inundación. Este segundo período reproductivo concordaría a la vez con los reportes de Campos (1970) para poblaciones diádromas en esta misma cuenca hidrográfica y con los ciclos gonádicos entregados por Peredo \& Sobarzo (1994) en el río Cautín (IX Región, 
Chile). Estos datos son consistentes también con las fechas reproductivas entregadas por Barriga et al. (2002) para poblaciones landlocked en Argentina. No obstante, Barriga et al. (2007) evidencian distintos valores de $K$ para poblaciones landlocked (residentes) de G. maculatus en el río Caleufú (norte de Patagonia, Argentina), puesto que el mayor incremento ocurre en primavera-verano y no en invierno, para posteriormente coincidir con el período de desove. Estos autores encuentran que los individuos adultos son los que mayormente aportan al incremento de $K$ durante el período de sobrelape entre cohortes, lo cual podría estar ocurriendo en las poblaciones analizadas aquí.
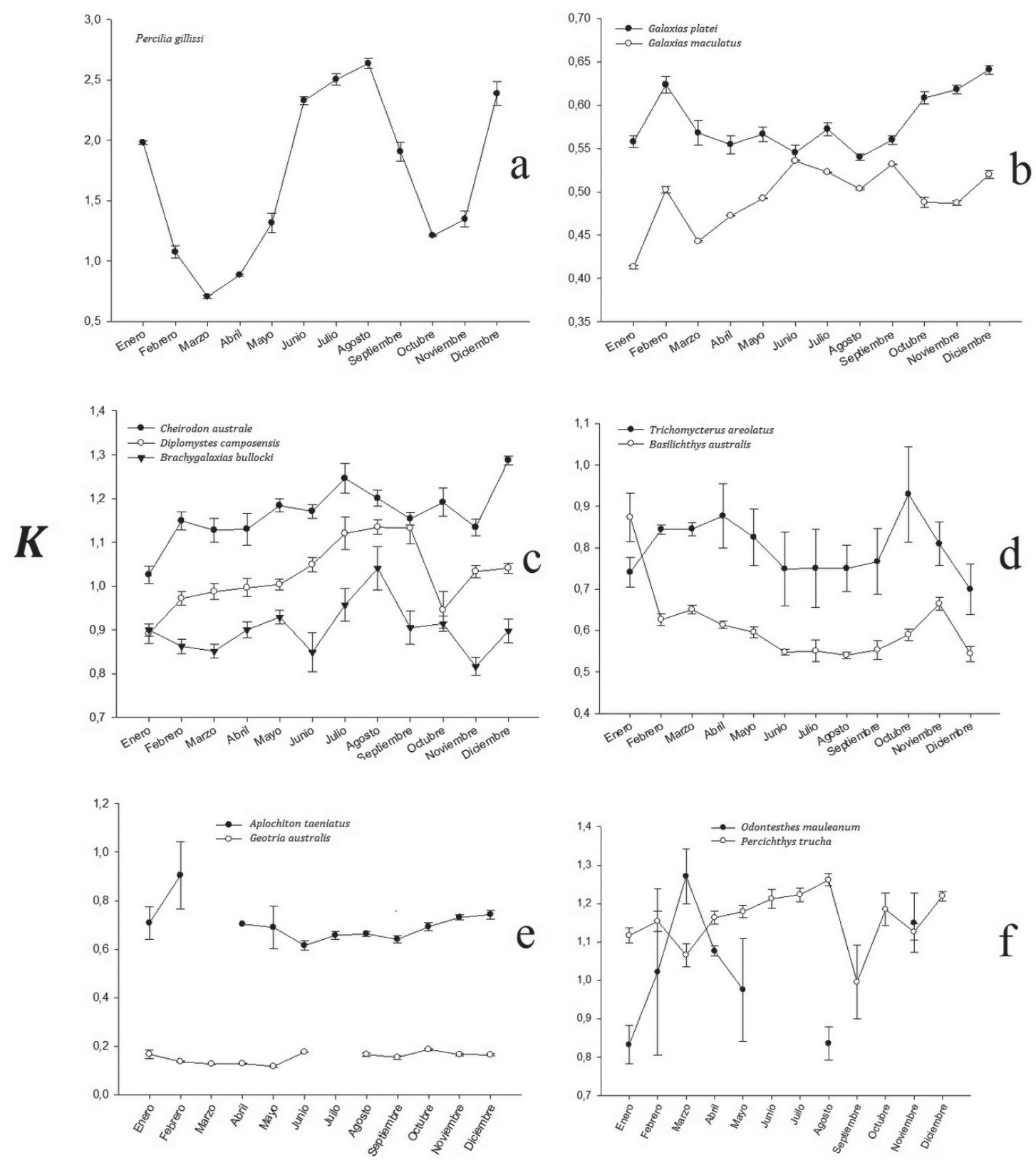

Figura 3. Variación mensual (media \pm ES) del factor de condición $(K)$ para ocho especies del río San Pedro; a) Percilia gillisi; b) Galaxias maculatus y Galaxias platei; c) Cheirodon australe, Diplomystes camposensis y Brachygalaxias bullocki; d) Trichomycterus areolatus y Basilichthys australis; e) Aplochiton taeniatus y Geotria australis; f) Odontesthes mauleanum y Percicthys trucha.

Figure 3. Monthly variation (mean $\pm \mathrm{SE}$ ) of condition factor $(K)$ for eight species from San Pedro River; a) Percilia gillisi; b) Galaxias maculatus and Galaxias platei; c) Cheirodon australe, Diplomystes camposensis and Brachygalaxias bullocki; d) Trichomycterus areolatus and Basilichthys australis; e) Aplochiton taeniatus and Geotria australis; f) Odontesthes mauleanum and Percicthys trucha. 
Los datos biológicos y reproductivos de la especie endémica $P$. gillissi son escasos. No obstante, las bruscas caídas de $K$ durante octubre (Fig. 3a) concuerdan con los reportes de Kilian \& Campos (1969) sobre un período de desove en primavera. Asimismo, Montoya et al. (2012) encuentran huevos durante finales de primavera y verano para este mismo sistema fluvial. Hacia marzo se detecta un decaimiento de $K$ asociado a la presencia de las mayores abundancias de juveniles (Montoya et al. 2012). Respecto del alza de $K$ observado durante invierno (Fig. 3a), registran mayores abundancias de tallas pequeñas durante julio. Por lo tanto, dicha alza estaría indicando principalmente la utilización de planicies de inundación por juveniles y reclutas para la alimentación y refugio.

Por otra parte, varios individuos de Ch. australe capturados en el río San Pedro superaron los $7 \mathrm{~cm}$ (LT) reportados como talla máxima por Ruiz \& Marchant(2004), alcanzando un ejemplar los 7,96 cm LT. Esta especie presenta una disminución de $K$ durante enero (Fig. 3c). Desafortunadamente, tampoco ha sido registrada evidencia directa sobre posturas para $C h$. australe. No obstante, se detectaron mayores frecuencias de postlarvas y juveniles hacia marzo en el mismo río (Montoya et al. 2012), indicando al menos una postura anual durante el verano. Similar período de desove mostraría la especie congenérica estrechamente relacionada (Dyer 2000) Ch. galusdae Eigenmann 1927, distribuída en Chile central (Ruiz \& Marchant 2004). Por otra parte, alzas de $K$ detectadas entre junio-agosto (Fig. 3c), estarían asociadas con el aprovechamiento de planicies de inundación para la alimentación de larvas y juveniles (Montoya et al. 2012) o probablemente con eventos de maduración gonadal en adultos, aunque esto último no ha sido reportado. Estudios realizados en Ch. interruptus (Jenyns, 1842) [especie introducida en aguas chilenas (Dyer 2000)], han evidenciado la potencialidad de reproducirse durante todo el año si las condiciones ambientales lo permiten (Menni \& Almirón 1994). Por lo que, si consideramos el aumento de caudal en julio y la consecuente mayor disponibilidad de alimento debido a la ocurrencia de planicies de inundación, se podría esperar un segundo evento de desove a fines de invierno para Ch. australe.

Así, algunas especies como G. maculatus, P. gillissi y Ch. australe comparten un patrón similar de variación de $K$ presentando dos máximos durante el año; un incremento progresivo durante el año hacia primavera-verano asociado a la maduración de los especímenes grandes, seguido de un descenso en enero asociado a la época de desove, y posteriormente otro incremento en $K$ durante la época invernal. Si bien, durante invierno (julio) podría encontrarse un acople de este patrón con el aumento de caudal, es probable que los ciclos reproductivos de estas especies estén relacionados también al aumento de las temperaturas o con la influencia e incremento del fotoperíodo, i.e. G. maculatus (sensu Campos 1970), propiciando una extensión de los ciclos reproductivos para estas especies durante el año.

En tanto $K$ de $G$. platei, tuvo un máximo a comienzos del verano (diciembre). Sin embargo, no existen antecedentes de los períodos reproductivos y fechas de postura que nos permitan asociar este máximo a un estado pre-desove de los individuos. Barriga et al. (2002) señalan un período de pre desove para el mes de diciembre en poblaciones de lagos patagónicos Argentinos. Cussac et al. (2004) señala la presencia de larvas para la época estival en lagos argentinos. Este hecho podría dar señal de un máximo de $K$ relacionado a la "maduración" de gónadas durante verano. No obstante, uno de los datos relevantes entregado por Barriga et al. (2002) es que sólo individuos capturados en profundidad, sobre 30 metros, en estos lagos se encontraban "maduros". Por ende, no poseemos antecedentes que nos permitan señalar concluyentemente que $G$. platei puede también reproducirse en ambientes lóticos, por lo cual es también probable que el máximo valor de $K$ para esta especie sea producto de una respuesta a una mayor disponibilidad de alimentos posterior a la época de deshielos de primavera.

Para B. australis, la variación de $K$ anual representa en mayor grado a individuos juveniles (98\%), ya que la longitud a la cual los individuos se encuentran maduros sexualmente es alrededor de $18 \mathrm{~cm}$ LT (Ruiz \& Marchant 2004). Por ende, la variación anual se relaciona en mayor grado al estado nutricional de los individuos. La variación mostró dos puntos altos, el mayor se observa en el mes de enero seguido de un máximo menor en el mes de noviembre (Fig. 3d). Este segundo punto alto coincide con la época de pre-desove señalada para algunas poblaciones de la zona centro sur de Chile, entre agosto y diciembre (Vila et al. 1981). Esto último, se relaciona a que los individuos de mayor tamaño $(N=8)$ incluidos en este estudio fueron capturados en el mes de noviembre y presentaron elevados valores de $K$.

Una de las especies que presentó baja frecuencia de captura fue $O$. mauleanum, a la vez con una muy baja presencia de individuos adultos en la muestra $(N>20 \mathrm{~cm} \mathrm{LT=6).} \mathrm{Para}$ estos individuos, sus valores de $K$ rodearon el valor 1,2. Similar a $B$. australis, esta especie presenta un máximo de $K$ en el mes de noviembre. Esto es coincidente con lo señalado por Klink \& Eckmann (1985) en relación a que esta especie se reproduce entre octubre y febrero en la cuenca del río Valdivia, por lo cual es probable que estos individuos capturados se encontraran en un estadío gonadal pre-desove.

Aplochiton taeniatus presentó sólo individuos juveniles de hasta 9,8 cm de LE, además de poca variación de $K$ para los 11 meses representados. Lattuca et al. (2008) señalan como 
talla mínima de madurez sexual para A.Zebra a las hembras de $14,2 \mathrm{~cm}$ LE, por lo que se espera que estas variaciones se deban a cambios en el estado nutricional de los individuos.

Por otro lado, especies como D. camposensis, B. bullocki y $P$. trucha mostraron sólo un incremento de $K$ al año, en época invernal de alto caudal, asociado con el desarrollo reproductivo, el cual culminaría en un evento de desove con una consecuente baja de $K$ hacia la segunda mitad del año. Si bien para $D$. camposensis no existen datos de postura, para la especie congénere $D$. nahuelbutaensis es concordante con los caudales más bajos y temperaturas altas del río, entre verano y otoño (Vila et al. 1996; Lundberg et al. 2004; Habit 2005). Por ende, D. camposensis podría utilizar los períodos de altos caudales para alimentarse, madurar y posteriormente reproducirse, de tal modo que un período de bajos niveles de $K$ (septiembre a noviembre) podría asociarse a eventuales desoves, y la presencia de reclutas en verano (enero-febrero) en el río San Pedro sería la evidencia final del ciclo de reproducción anual de la especie. Por otro lado, el estado de condición de B. bullocki, decrece bruscamente en noviembre, lo cual tendría sentido con el término de un período reproductivo, el cual ha sido descrito entre julio y octubre (Campos 1972). Este mismo patrón lo encontramos en $P$. trucha con un aumento paulatino de $K$ durante el invierno y un brusco descenso en primavera, este último período ha sido descrito como de maduración sexual para poblaciones en lagos argentinos (Buria et al. 2007). Sin embargo, como los rangos de tallas presentes en el río han sido considerados juveniles y no se encontraron adultos maduros, esta baja de $K$ podría relacionarse con una migración de los individuos más robustos de la población del río San Pedro hacia el lago Riñihue, para completar su período de maduración.

Otras especies como T. areloatus no evidenciaron un claro patrón de variación en su factor de condición intranual, pues sus valores de $K$ presentaron una alta variación intrames (entre abril-diciembre). Es posible que estos niveles tan altos de variación intra-mes puedan ser explicados por la presencia de una segunda especie de tricomictérido coexistiendo en simpatría con $T$. areolatus, pero que no había sido identificada anteriormente por su gran similitud morfológica, siendo actualmente diferenciadas por un único carácter diagnostico externo. Específicamente, Hatcheria macraei (Girard 1855), que hasta hace poco consideraba una distribución geográfica mayoritariamente trasandina y únicamente en Patagonia para el territorio nacional, fue registrada hasta la cuenca del río Imperial incluyendo la cuenca del río Valdivia (Unmack et al. 2009). Los registros de esta especie en cuencas chilenas evidencian poblaciones muy reducidas y escasas, lo que eventualmente podría haber alterado los resultados presentados aquí al haber considerado una sola especie. Dado que en alopatría ambas especies se muestran abundantes, es posible que exista competencia en cuanto al uso de recursos (sensu Unmack et al. 2009), y por ende, que presenten diferenciación espacio-temporal en sus patrones de crecimiento y estrategias reproductivas.

El factor de condición es útil para comparar cambios estacionales del estado nutricional de los individuos de una población (Blackwell et al. 2000; Froese 2006). Sin embargo, su análisis requiere ser complementado con otras herramientas de observación directa (e.g. análisis dietarios, análisis de actividad gonadosómica, seguimiento de cohortes, etc.) (McPherson et al. 2011). Por tal motivo, para alcanzar un entendimiento acabado de la dinámica y los ciclos de vida de los peces nativos del país, se hace perentorio realizar mayores esfuerzos de investigación enfocados en los aspectos biológicos de estas especies.

\section{CONCLUSIONES}

Uno de los aspectos más relevantes del presente estudio, es que entrega datos inéditos sobre las características de las poblaciones de peces nativos en un seguimiento de largo plazo (29 meses equivalentes a $c a$. de 2,5 ciclos anuales). Por otra parte, fueron dispares los resultados obtenidos para las distintas especies en relación al tipo de crecimiento y a la variación de $K$ a través del año, evidenciando distintos patrones y estrategias de crecimiento y reproducción. Un grupo de estas especies estarían más asociadas al aumento de caudal del río, en tanto que otras al aumento de las temperaturas y fotoperíodo del mismo. Para la totalidad de las especies analizadas fue posible encontrar estadíos juveniles, por lo cual la comunidad de peces nativos del río San Pedro constituye un importante centro de desarrollo y reclutamiento de especies residentes como de especies migratorias. Dadas estas características, se torna imprescindible considerar tales registros para la evaluación de futuros proyectos de impacto ambiental asociados a la cuenca.

\section{AGRADECIMIENTOS}

Agradecemos a COLBUN S.A. por financiar este estudio y al proyecto DIUC Semilla Patagonia 210.310.057-1SP por el financiamiento de la publicación.. A los propietarios de los predios aledaños al río por permitirnos ingresar a ellos, y muy especialmente al Hotel Riñimapu por las facilidades brindadas para llevar a cabo esta investigación. Igualmente se agradece a todas las personas que directa o indirectamente participaron en la elaboración de este estudio. Finalmente 
se agradece al proyecto Fondecyt 1110441 que permitió la finalización de este manuscrito.

\section{REFERENCIAS}

Agboola, J.I.\&AneteKhai, M.A. 2008. Length-weightrelationships of some fresh and brackish water fishes in Badagry Creek, Nigeria. Journal Of Application Ichthyology 24:623-625.

Arismendi, I., Penaluna, B. \& Soto, D. 2011. Body condition indices as a rapid assessment of the abundance of introduced salmonids in oligotrophic lakes of southern Chile. Lake and Reservoir Management 27:61-69.

Barriga J., Battini, M., Macchi, P., Milano D. \& Cussac, V. 2002. Spatial and temporal distribution of landlocked Galaxias maculatus and Galaxias platei (Pisces: Galaxiidae) in a lake in the South American Andes. New Zealand Journal of Marine and Freshwater Research 36:345-359.

Barriga, J.P., Battini, M.A. \& Cussac, V.E. 2007. Annual dynamics variation of a landlocked Galaxias maculatus (Jenyns 1842) population in a Northern Patagonian river: occurrence of juvenile upstream migration. Journal of Application Ichthyology 23:128-135.

Blackwell, B., Seamans, T., Helon, D. \& Dolbeer, R. 2000. Early loss of Herring Gull glutches after egg-oiling. Wildlife Society Bulletin 28(1):70-75.

Buria, L., Walde, S., Battini, M., Macchi, J., Alonso, M., Ruzzante, D \& Cussac, V. 2007. Movement of a South American perch Percichthys trucha in a mountain Patagonian lake during spawning and prespawning periods. Journal of Fish Biology 70:215-230.

Campos, H. 1970. Galaxias maculatus (Jenyns) en Chile, con especial referencia a su reproducción. Boletín del Museo Nacional de Historia Natural de Chile 31:5-20.

CAmpos, H. 1972. Breeding season and early development of Brachygalaxias bullocki Osteichthyes: Galaxiidae). The Texas Journal of Science 23(4):531-544.

Carlander, K.D. 1969. Handbook of freshwater fishery biology. University Press, The Iowa State, EEUU. Vol.I 752 pp.

Chapman, A., Morgan, D., Beatty, S. \& Gill, H. 2006. Variation in life history of land-locked lacustrine and riverine populations of Galaxias maculatus (Jenyns 1842) in Western Australia. Environmental Biology of Fish 77:2137.

Cussac V., Ortubay, S., Inglesias, G., Milano, D., Lattuca, M., Barriga, J., Battini, M. \& Gross, M. 2004. The distribution of South American galaxiid fishes: the role of biological traits and post-glacial history. Journal of Biogeography 31: 103-121.

DYER, B. 2000. Systematic review and biogeography of the freshwater fishes of Chile. Estudios Oceanológicos 19:7798.

Froese, R. 2006. Cube law, condition factor and weight-length relationships: history, meta-analysis and recommendations. Journal of Applied Ichthyology 22:241-253.

Granado, C. 1996. Ecología de peces. Secretariado de Publicaciones de la Universidad de Sevilla. Sevilla, España. 353 pp.

Habit, E. 2005. Aspectos de la biología y hábitat de un pez endémico de Chile en peligro de extinción (Diplomystes nahuelbutaensis Arratia, 1987). Interciencia 30:8-11.

Habit, E., Victoriano, P. \& CAmpos, H. 2005. Ecología trófica y aspectos reproductivos de Trichomycterus areolatus (Pisces, Trichomycteridae) que colonizan ambientes lóticos artificiales. Revista Biología Tropical 52(4):195-210.

Habit, E., Dyer, B. \& Vila, I. 2006. Estado de conocimiento de los peces dulceacuícolas de Chile: estado de su conocimiento. Gayana 70(1):100-112.

Killian, E. \& Campos, H. 1969. Susswasserschwamme als ortder Brutpflege eipe Fisches. Naturwissenschaften 56(5):333334.

Klink, A. \& Eckmann, R. 1985. Age and growth, feeding habits, and reproduction of Cauque mauleanum (Steindachner 1896) (Pisces: Atherinidae) in southern Chile. Studies Neotropical Fauna \& Environment 20(4):239-249.

Lattuca, M. E., Brown, D., Castimeira, L., Remzi, M., Luizom, C., Urbamski, J. \& Cussac, V. 2008. Reproduction of landlocked Aplochiton zebra Jenyns (Pisces, Galaxiidae). Ecology of Freshwater Fish 17(3):394-405.

Lundberg J., T., Berra, M., Tim, J., Friel \& P., John. 2004. First description of small juveniles of the primitive catfish Diplomystes (Siluriformes: Diplomystidae). Ichthyologial Exploration of Freshwaters 15(1):71-82.

McCallum, H. 2000. Population Parameters: Estimation for Ecological Models. Blackwell Science Ltd. Oxford, London. 348 pp.

McPherson, L.R., Slotte, A., Kvamme, C., Meier, S. \& Marshall, C.T. 2011 Inconsistencies in measurement of fish condition: a comparison of four indices of fat reserves for Atlantic herring (Clupea harengus). ICES Journal of Marine Science 68: 52-60.

Menni, R.C. \& Almirón, A.E. 1994. Reproductive seasonality in fishes manmade ponds in temperate South America. Neotropica 40(103-104):75-85.

Montoya, G., Jara, A., Solis-Lufí, K., Colin, N. \& Habit, e. 2012. Primeros estadios del ciclo de vida de peces nativos del río San Pedro (Cuenca del río Valdivia, Chile). Gayana 76 (Número Especial): 86-100.

Peredo, S. \& Sobarzo, C. 1994: Actividad gonádica estacional de Galaxias maculatus (Jenyns, 1842) en el río Cautín, IX Región, Chile. Boletín de la Sociedad de Biología de Concepción 65:65-70.

RenNie, M.D. \& Verdon, R. 2008. Evaluation of condition indices for the lake whitefish, Coregonus clupeaformis. North American Journal of Fisheries Management 28:12701293.

RickeR, W. 1975. Computation and interpretation of biological statistics of fish populations. Bulletin Fisheries Research. Board of Canada. 191: 382 pp.

Ruiz, V.H. \& Marchant, M. 2004. Ictiofauna de aguas continentales de Chile. Facultad de Ciencias Naturales y Oceanográficas, Universidad de Concepción, Chile. 356 pp.

Treer, T., Sprem, N.,Torcu-Koc, H., Sun, Y. \& Piria, M. 2008. Length-weight relationships of freshwater fishes of Croatia. Journal of Application Ichthyology 24:626-628.

Trudel, M., Tucker, S., Morris, J., Higgs, D. \& Welch, D. 2005. Indicators of energetic status in juvenile coho and chinook salmon. North American Journal of Fisheries Management 
25:374-390.

Unmack, P., Habit, E. \& Johnson, J.P. 2009. New records of Hatcheria macraei (Siluriformes, Trichomycteridae) from Chilean Province. Gayana 73(1):102-110.

Vila, I., Soto, D. \& Bahamondes, I. 1981. Age and growth of Basilichthys australis Eigenmann 1927 in Rapel reservoir,
Chile. (Pisces, Atherinidae). Studies Neotropical Fauna \& Environment 16(1):9-22.

Vila, I., Contreras, M. \& Fuentes, L. 1996. Reproducción de Diplomystes nahuelbutensis Arratia, 1987 (Pises: Diplomystidae). Gayana Oceanología 4:129-137.

Recibido: 02.01 .12

Aceptado: 27.02.12 